\title{
Sensibilidad y especificidad de la Escala de Alvarado en el diagnóstico de pacientes atendidos por apendicitis aguda en Hospitales del Cusco
}

\author{
Meléndez Flores Jhuvitza Estela* 1,a; Cosio Dueñas Herbert 2,3,b,c; William Senen Sarmiento Herrera ${ }^{2, a, d}$
}

RESUMEN

Objetivo: Determinar la sensibilidad y especificidad de la Escala de Alvarado para el diagnóstico de pacientes atendidos con apendicitis aguda en Hospitales del Cusco durante el periodo 2011 al 2016.

Materiales y métodos: Estudio de tipo descriptivo, transversal y observacional. Se revisaron las historias clínicas de 316 pacientes atendidos por apendicitis aguda durante el periodo 2011 al 2016 en el servicio de Cirugía de tres hospitales de la ciudad del Cusco. Con un muestreo no probabilístico por conveniencia, la variable Escala de Alvarado se definió mediante la valoración clínica y el análisis histopatológico fue el Gold estándar de diagnóstico. Se usó el paquete estadístico SPSS 22 en español y se analizó la variable con pruebas de sensibilidad y especificidad,se establecieron valores predictivos positivos y negativos por lo que se usó el análisis de curvas ROC (receiver operating characteristic curve).

Resultados: La edad promedio del estudio fue 34 años. El 55,7\% de los pacientes fueron varones y el tiempo promedio de enfermedad fue 16 horas. De la evaluación total, en el 13,6 \% se descartó el diagnóstico de apendicitis aguda por estudio histopatológico; de este grupo 11,9\% eran hombres y 15,7\%, mujeres. En el análisis la Escala de Alvarado presentó una sensibilidad del $78 \%$ y especificidad de $44,2 \%$ valor predictivo positivo de $89,8 \%$ y valor predictivo negativo de $13,3 \%$. El área bajo la curva ROC fue de 61,1\%.

Conclusiones: La Escala de Alvarado tuvo un alto porcentaje de sensibilidad y una buena especificidad en relación al diagnóstico histopatológico.

Palabras clave: Apendicitis; Sensibilidad y especificidad; Confiabilidad y validez (Fuente: DeCS BIREME).

\section{Sensitivity and specificity of the Alvarado Score in the diagnosis of patients treated for acute appendicitis in hospitals of Cusco}

\section{ABSTRACT}

Objective: To determine the sensitivity and specificity of the Alvarado Score for the diagnosis of patients with acute appendicitis treated in hospitals of Cusco during the period from 2011 to 2016.

Materials and methods: A descriptive, cross-sectional and observational study. The medical records of 316 patients treated for acute appendicitis during the period from 2011 to 2016 in the Surgery Department of three hospitals in the city of Cusco were reviewed. With a non-probabilistic convenience sampling, the Alvarado Score variable was defined by clinical assessment, and the histopathological analysis was the diagnostic gold standard. The SPSS Statistics 22.0 was used, and the variable was analyzed with sensitivity and specificity tests. Positive and negative predictive values were established, and the receiver operating characteristic $(\mathrm{ROC})$ curve analysis was used.

Results: The average age was 34 years, $55.7 \%$ were male, and the average disease duration was 16 hours. Out of the total evaluation, $13.6 \%$ of the patients had a histopathological diagnosis ruling out acute appendicitis, from which $11.9 \%$ were males and $15.7 \%$ were females. The Alvarado Score presented a sensitivity of $78 \%$, specificity of $44.2 \%$, positive predictive value of $89.8 \%$, and negative predictive value of $13.3 \%$. The area under the ROC curve was $61.1 \%$.

Conclusions: The Alvarado Score had a high percentage of sensitivity and a good specificity regarding the histopathological diagnosis.

Keywords: Appendicitis; Sensitivity and specificity; Reliability and validity (Source: MeSH NLM)

1. Universidad Andina del Cusco. Cusco, Perú.

2. Universidad Peruana Cayetano Heredia. Lima, Perú.

3. Universidad Católica de Santa María. Arequipa, Perú.

a. Médico Cirujano.

b. Cirujano Dentista.

c. Doctor en Educación, Magíster en Estomatología.

d. Magister en Educación.

* Autor corresponsal. 


\section{INTRODUCCIÓN}

El dolor abdominal es motivo de consulta frecuente en el área de urgencias de los establecimientos de salud, se estima en porcentajes por encima del 5,1\%; es decir, uno de cada 20 pacientes que acuden al servicio de urgencias presenta este síntoma. En su reporte de investigación Peralta, Caballero y Mora manifiestan que el dolor abdominal, como motivo de consulta en los establecimientos de urgencia, representa entre el 5 al $10 \%$ del total de consultas ${ }^{(1)}$. Por otro lado, el médico debe de reunir las competencias necesarias para reconocer las características de la misma y poder tomar la decisión más apropiada y oportuna para el paciente, ya que el $23,3 \%$ de los pacientes con dolor abdominal terminaron sometidos a procedimientos quirúrgicos ${ }^{(2)}$. Un estudio en Cuba ha demostrado que en el $28 \%$ de las urgencias quirúrgicas los pacientes adultos son apendicectomizados. Así mismo, en muchos países latinoamericanos la apendicitis aguda es la principal causa de procedimientos quirúrgicos de la región abdominal (3). En Argentina, se estima que el $7,6 \%$ de la población ha sufrido apendicectomía, de dicho porcentaje, la apendicectomía negativa podría llegar a no menos del $20 \%{ }^{(4)}$.

La apendicitis aguda que no ha recibido asistencia médica apropiada puede ser causa de muerte, por lo que es responsabilidad del personal médico centrarse en sospechas diagnósticas sin dejar pasar por alto estas dolencias ${ }^{(3,5)}$. El diagnóstico de la apendicitis aguda es fundamentalmente clínico, mediante los exámenes físicos, la experiencia (a mayor experiencia mayor certeza de diagnóstico) y el uso de exámenes complementarios. Es una de las cirugías de urgencia más frecuentes $(4,6)$.

La tarea del médico de urgencias es tomar la mejor decisión clínica, en el menor tiempo y con el menor costo posible; para tal fin se han hecho esfuerzos en investigación para aumentar la certeza del diagnóstico de la apendicitis aguda, con base en los hallazgos clínicos, laboratoriales y por imágenes; así mismo, se han desarrollado sistemas de puntuación clínica para orientar al médico en un diagnóstico correcto, con el fin de disminuir las apendicectomías negativas ${ }^{(6)}$.

La apendicitis se caracteriza por presentar síntomas como el dolor abdominal que migra a la fosa iliaca derecha, dolor en el cuadrante inferior derecho, dolor al rebote o descompresión, acompañado de fiebre, anorexia, náuseas y, a veces, vómito. Los resultados de exámenes de laboratorio como leucocitosis y desviación a la izquierda, fueron incluidos en diferentes escalas o test que permiten el diagnóstico de apendicitis aguda, se toma como base la parte clínica y complemento la parte laboratorial sencilla (3). El test o escala de Alvarado propuesta por el mismo autor en 1986, para el diagnóstico de apendicitis aguda, considera estas ocho características principales extraídas del cuadro clínico. Los signos y síntomas considerados importantes son la migración del dolor, anorexia, náuseas y/o vómitos, neutrofilia mayor al $75 \%$, rebote, elevación de la temperatura mayor a $38{ }^{\circ} \mathrm{C}$, defensa en el cuadrante inferior derecho y leucocitosis. Los seis primeros con una valoración de 1 punto y los dos últimos con una valoración de 2 por síntoma, lo que hace un total de diez puntos. El uso de la escala de Alvarado permite clasificar a los pacientes que acuden al servicio de urgencias con dolor abdominal en la fosa iliaca derecha en tres grupos: riesgo bajo (0-4 puntos) con probabilidad baja de apendicitis, riesgo intermedio (5-7 puntos) probabilidad promedio de apendicitis, riesgo alto (8-10 puntos) alta probabilidad de apendicitis, estos últimos deben de ser sometidos a cirugía de inmediato ${ }^{(6)}$. Los criterios de Alvarado son, probablemente, el sistema de mayor difusión y una herramienta útil para el diagnóstico de apendicitis aguda que podemos aplicar en todos los pacientes con dolor abdominal agudo que sugiera una inflamación del apéndice. La escala de Alvarado es la más aceptada por los servicios de urgencias en todo el mundo, con una sensibilidad de $68 \%$ y especificidad de $87,9 \%^{(5,7)}$.

Hernández y Domínguez hacen una descripción de los criterios diagnósticos propuestos por Alvarado, y observan que el criterio más frecuente fue el dolor en la fosa iliaca derecha, en el $100 \%$ de pacientes intervenidos quirúrgicamente, y el rebote, el más específico (94,3\% de los casos). Otros criterios frecuentes fueron las náuseas y los vómitos $(72,5 \%)$, leucocitosis $(85 \%)$, dolor migratorio $(6,5 \%)$, anorexia $(54,3 \%)$, neutrofilia $(84,3 \%)$ y fiebre $(48,7 \%){ }^{(7)}$.

La escala de Alvarado continua siendo una ayuda en el diagnóstico de la apendicitis aguda y una herramienta útil para mejorar la oportunidad y eficacia de la evaluación, sobre todo en los servicios donde no se cuente con estudios por imágenes ${ }^{(8,9)}$. Sanabria et al. evidenciaron una disminución del $10 \%$ de los costos y $8 \%$ de las apendicectomías no terapéuticas, así como la reproductibilidad por parte de los médicos del área de urgencias, disminuyendo significatvamente los casos de apendicectomías innecesarias ${ }^{(5)}$. Ricci et al. concluyen que los criterios de la escala de Alvarado reducen significativamente las apendicectomías negativas ${ }^{(4)}$. 
La certeza diagnóstica en esta entidad es todavía un reto, a pesar de las múltiples herramientas que se han diseñado para su determinación diagnóstica en la etapa inicial en la que los métodos prácticos clínicos son los más empleados. Las puntuaciones diagnósticas de apendicitis aguda han demostrado su valor cuando se incorporan en la práctica clínica rutinaria ${ }^{(10)}$.

En los últimos años, el uso de las imágenes para la valoración de la apendicitis aguda, ha ido en aumento, a través del ultrasonido, la resonancia y de la tomografía axial computarizada (TAC), por lo que se han convertido en una práctica común, sobre todo en los centros donde se cuenta con estas herramientas. Permiten el diagnóstico de apendicitis, con una sensibilidad de $94 \%$ y especificidad de $98 \%$ y, de esta forma, se reduce significativamente el nivel de apendicitis negativa ${ }^{(7)}$. También se cuenta con el ultrasonido, que es la mejor prueba auxiliar de imágenes para el diagnóstico: se evidencia que cuando se indica negatividad para la apendicitis aguda la posibilidad que esté presente es de $17 \%{ }^{(8)}$. Dichos medios de evaluación no están disponibles, ni se utilizan en todas las instituciones, por sus elevados costos operativos ${ }^{(4)}$.

\section{MATERIALES Y MÉTODOS}

Diseño y población de estudio

Se realizó un estudio de tipo descriptivo, transversal y observacional, en el que se incluyeron 316 historias clínicas de pacientes atendidos por probable apendicitis aguda durante el periodo 2011 al 2016 en los servicios de cirugía de los hospitales Nacional Adolfo Guevara Velasco, Regional del Cusco y Antonio Lorena. El objetivo fue determinar la sensibilidad y especificidad del uso de la escala de Alvarado en el diagnóstico de la apendicitis aguda, y compararlo con el diagnóstico histopatológico.

Variables y mediciones

Las variables evaluadas fueron la edad, sexo, tiempo de evolución previo a la cirugía, el valor diagnóstico de Alvarado y el diagnóstico histopatológico. Este último se definió como apéndice normal y como apéndice con cambios $o$ apendicitis. A todos los pacientes operados se les realizó la valoración clínica y se les clasificó según la escala de Alvarado (dato registrado en la historia clínica); así mismo, el reporte histopatológico con el diagnóstico definitivo como el estándar de oro. Los criterios de Alvarado fueron ocho: náuseas y/o vómitos, cronología de Murphy, hiporexia, fiebre, dolor en la fosa iliaca derecha, reacción peritoneal localizada (signo de Blumberg), leucocitosis y desviación a la izquierda.
Cada síntoma recibió una puntuación estandarizada, y llegan a sumar 10 puntos; cuando el valor fue mayor a 7 se estableció riesgo alto de apendicitis.

\section{Análisis estadístico}

El análisis estadístico fue descriptivo para las características generales de la población y las variables de interés se sometieron a pruebas de sensibilidad y especificidad; se establecieron valores predictivos positivos y negativos. Se valoró la utilidad de la escala de Alvarado para el diagnóstico de apendicitis aguda mediante el análisis de curvas ROC. Se usó el paquete estadístico SPSS 22 en español.

\section{Consideraciones éticas}

Se tuvieron en consideración los aspectos éticos planteados en la Declaración de Helsinki, referente a la investigación en humanos. Para ello se tomaron en cuenta los principios de beneficencia, no maleficencia, autonomía y justicia. Se mantuvo una estricta confidencialidad en cuanto a la divulgación de la información.

\section{RESULTADOS}

De la muestra obtenida con diagnóstico probable de apendicitis aguda (AA), se encontraron 140 mujeres $(44,3 \%)$ y 176 hombres (55,7\%). La edad media de los pacientes fue de 34 años con una desviación estándar (DE) de 14 años, un rango de 11 a 81 años. El promedio del tiempo de evolución previo a la cirugía fue de 16 horas con una DE de 8 horas, un rango de 3 a 30 horas de espera.

El diagnostico histopatológico de apendicitis aguda se confirmó en 273 pacientes $(86,4 \%)$ y se descartó en 43 pacientes $(13,6 \%)$. El diagnóstico temprano de apendicitis aguda mediante la escala de Alvarado, practicado antes de la intervención quirúrgica, se confirmó en 237 pacientes (75\%) y se descartó en 79 pacientes ( $25 \%$ ) (Tabla 1$)$.

La validez de la escala de Alvarado se evaluó mediante la sensibilidad y la especificidad que tienen como función juzgar la prueba, se obtuvo una sensibilidad de $78 \%$ y una especificidad 44,2 \%. Para determinar la capacidad de diagnosticar o descartar la presencia de enfermedad se obtuvo valor predictivo positivo de $89,8 \%$ y el valor predictivo negativo de $24 \%$ (Tabla 2).

Se realizó una curva ROC para la escala de Alvarado, y se obtuvo un área bajo la curva de 0,611 (Figura 1). Al emplear este análisis estadístico se encontró que el punto de corte $>5$ es el de mayor precisión diagnóstica. 
Tabla 1. Diagnóstico temprano de apendicitis aguda (AA) con la escala de Alvarado y el diagnóstico histopatológico

\begin{tabular}{|c|c|c|c|c|c|}
\hline & & & \multicolumn{2}{|c|}{ Escala de Alvarado } & \multirow[t]{2}{*}{ Total } \\
\hline & & & $\begin{array}{l}\text { Sí diagnóstico } \\
\text { temprano de AA }\end{array}$ & $\begin{array}{l}\text { NO diagnóstico } \\
\text { temprano de AA }\end{array}$ & \\
\hline \multirow[t]{2}{*}{$\begin{array}{l}\text { Diagnóstico } \\
\text { histopatológico }\end{array}$} & Sí AA & $\begin{array}{l}\text { Recuento } \\
\% \text { del total }\end{array}$ & $\begin{array}{r}213 \\
67,4 \%\end{array}$ & $\begin{array}{r}60 \\
19,0 \%\end{array}$ & $\begin{array}{r}273 \\
86,4 \%\end{array}$ \\
\hline & No AA & $\begin{array}{l}\text { Recuento } \\
\% \text { del total }\end{array}$ & $\begin{array}{r}24 \\
7,6 \%\end{array}$ & $\begin{array}{r}19 \\
6,0 \%\end{array}$ & $\begin{array}{r}43 \\
13,6 \%\end{array}$ \\
\hline Total & & $\begin{array}{l}\text { Recuento } \\
\% \text { del total }\end{array}$ & $\begin{array}{r}237 \\
75,0 \%\end{array}$ & $\begin{array}{r}79 \\
25,0 \%\end{array}$ & $\begin{array}{r}316 \\
100,0 \%\end{array}$ \\
\hline
\end{tabular}

Cruce de variables para ver porcentajes de diagnósticos tempranos con la escala de Alvarado y el diagnóstico histopatológico.

Tabla 2. Valores diagnósticos de la escala de Alvarado en pacientes intervenidos quirúrgicamente con diagnóstico probable de apendicitis aguda

\begin{tabular}{|l|l|}
\hline & Escala de Alvarado \\
\hline Sensibilidad & $78,0 \%$ \\
\hline Especificidad & $44,2 \%$ \\
\hline Valor predictivo positivo & $89,8 \%$ \\
\hline Valor predictivo negativo & $24,0 \%$ \\
\hline
\end{tabular}

*Porcentajes de confiabilidad y validez de la escala de Alvarado.

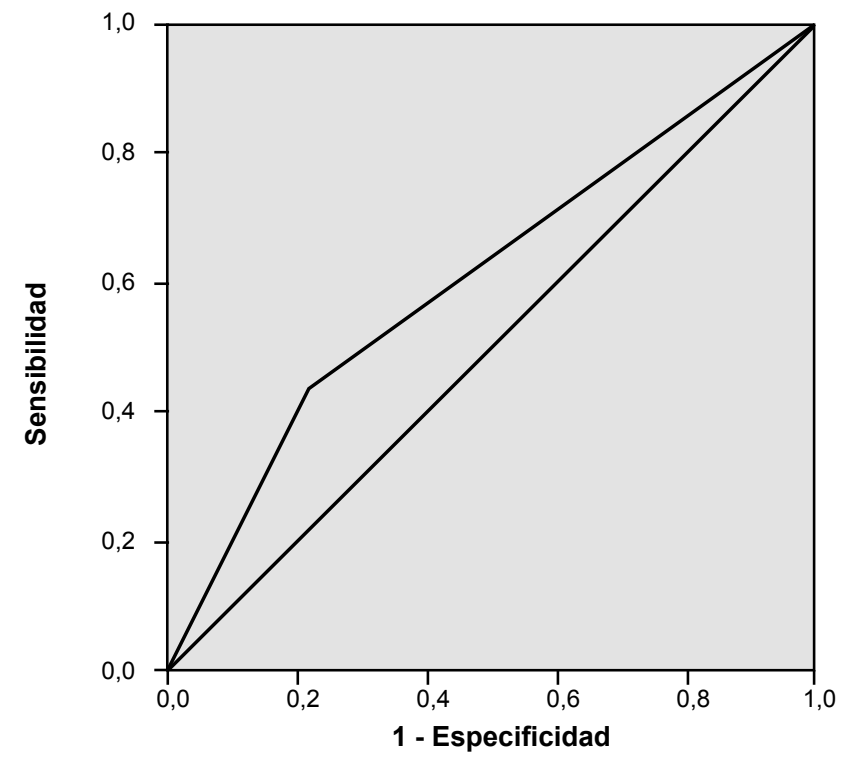

Figura 1. Gráfico de curva ROC del test de Alvarado (área bajo la curva es de 0,611), con diagonal de referencia

\section{DISCUSIÓN}

Dentro del servicio de emergencia y cirugía general el diagnóstico temprano de apendicitis aguda es muy importante para evitar complicaciones que pongan en riesgo la vida del paciente, prolongar la estancia hospitalaria y elevar los costos de hospitalización. La escala de Alvarado se presenta como una alternativa consistente en el registro de ocho características principales de los signos y síntomas importantes de esta enfermedad, 
con valoraciones que permitan sugerir la intervención inmediata del paciente. A todas las historias clínicas de los pacientes atendidos por apendicitis aguda se les realizó la valoración clínica mediante la escala de Alvarado. Se consideró positivo a todo paciente con puntuación mayor a 7, valor que nos indica la probabilidad de apendicitis. Durante la recolección de los datos se descartaron algunas historias clínicas como unidades de análisis porque no consignaban la información completa y presentaban una grafía incomprensible.

Se estima que el riesgo de padecer apendicitis aguda en algún momento de la vida es mayor en el sexo masculino, Reyes et al. en el 2012 evidencian que el $8,6 \%$ se presentó en varones y $6,7 \%$ en mujeres ${ }^{(6)}$. A toda nuestra muestra se aplicó la escala de Alvarado y se encontró el $44,3 \%$ (140) mujeres y 55,7 \% (176) hombres, pacientes valorados por el servicio de cirugía general de los tres centros hospitalarios de la ciudad del Cusco. De todos los antecedentes revisados, fueron los hombres quienes presentaron una mayor frecuencia de este cuadro clínico, Cedillo et al. encontraron 55 hombres y 44 mujeres ${ }^{(8)}$. Hernadez y Dominguez hallaron un $53,3 \%$ de hombres y $46,6 \%$ de mujeres ${ }^{(7)}$. Opina et al. obtuvieron que el $56,6 \%$ de los pacientes apendicectomizados fueron hombres ${ }^{(9)}$. Ricci halló $50,6 \%$ de hombres y $49,4 \%$ de mujeres ${ }^{(4)}$. Reyes et al. encontraron $51,4 \%$ en hombres y $48,6 \%$ en mujeres ${ }^{(6)}$. Resultados que difieren con los hallazgos de De Quesada et al. quienes encontraron un predomino en las mujeres con el $52 \%$, frente al $48 \%$ en los hombres ${ }^{(3)}$, así como lo hallado por Peralta quien reporto un $57 \%$ de mujeres y $43 \%$ de hombres ${ }^{(1)}$.

La población que mayormente ha sido sometida a intervenciones por apendicitis aguda, se encuentra entre los 25 a 35 años. En nuestro estudio el rango de edad fue muy variado en los pacientes sometidos a una apendicectomía, se encontraron pacientes de 11 años hasta los 81 años, con una media de 34 años, resultados similares a lo hallado por Peralta que encontró una media de 30,5 años ${ }^{(1)}$. Hernández y Domínguez que encontraron una media de 36,9 años con un rango de 14 a 89 años ${ }^{(7)}$, y lo hallado por De Quesada que encontró una media de 37,9 años ${ }^{(3)}$, Ricci et al. hallaron una población con una media de 40 años con un rango de 14 a 91 años ${ }^{(4)}$. Nuestros resultados difieren con lo hallado por Cedillo et al. que encontraron una población más joven con una media de 25,5 años, y un rango de edad de 14 a 60 años ${ }^{(8)}$.

Es común la consulta de pacientes con dolor localizado en la fosa iliaca derecha y síntomas asociados al cuadro de apendicitis aguda en el área de urgencias, lo que obliga un análisis exhaustivo por parte del profesional médico, que implica someter al pacientes a los exámenes de laboratorio $y$, en algunos casos, de imágenes para descartar otras dolencias. Todos estos procedimientos clínicos determinan una estancia o tiempo de evolución previo a la cirugía. En nuestro estudio la media fue de 16 horas con un rango de 3 a 30 horas. Promedio de estancia que difiere a casi todos los estudios comparados, De Quesada presentó presento un tiempo de evolución fue de 25 horas en promedio ${ }^{(3)}$. Cedillo et al. presento el tiempo de evolución prehospitalaria con una media de 40 horas ${ }^{(8)}$. Hernández y Domínguez presentaron un tiempo de evolución previo a la valoración médica de 40,9 horas, con un rango de 12 a 96 horas ${ }^{(7)}$. El diagnóstico temprano y certero disminuye la mortalidad y morbilidad, no obstante, el proceso diagnóstico constituye un desafío para el médico de urgencias, que tiene que lidiar con los procesos administrativos y el uso del área de laboratorio.

El diagnóstico correcto de apendicitis aguda varía sustancialmente entre diferentes reportes de investigación, que llegan a registrar hasta un 70 \% en los adultos (9). Alvarado propuso sus criterios para evaluar el síndrome de la fosa iliaca derecha, con el propósito de reducir el número de apendicectomías negativas ya que existe una alta posibilidad que muchos pacientes con síntomas abdominales sean operados con apéndices normales. Se hace el diagnóstico definitivo mediante el informe de anatomía patológica ${ }^{(4)}$. En nuestro estudio se encontró que el $13,6 \%$ de los pacientes intervenidos por apendicitis aguda tuvieron un apéndice normal, diagnóstico realizado histológicamente. Reyes et al. encontraron un índice de apendicitis negativa, histológicamente normal, en el $18,6 \%{ }^{(6)}$. De Quesada comprobó la enfermedad histológicamente en el $86 \%$ de pacientes operados y el $14 \%$ fueron pacientes operados con el apéndice normal o negativo, y encontraron que las cifras de pacientes con apéndices normales no difieren con lo revisado en otras investigaciones ${ }^{(3)}$. Ospina et al. Encontró una concordancia diagnóstica de $75,2 \%$ entre la escala de Alvarado y el resultado histopatológico ${ }^{(9)}$. Se estima que la frecuencia de apendicitis negativas en la población de mujeres en edad reproductiva puede aumentar de $20,7 \%$ a $26 \%{ }^{(4)}$. Se acepta que un cirujano con práctica habitual en su servicio pueda tener un $15 \%$ de apendicectomías negativas, cifra que pueden verse incrementada, en especial, en mujeres menores de 35 años ${ }^{(6)}$. En tal sentido, se hace imperativo, ante estos resultados, recurrir al diagnóstico por imágenes y al uso de exámenes de laboratorio más complejos, que en definitiva elevarían el costo de los tratamientos ${ }^{(3)}$.

La sensibilidad es la probabilidad de clasificar correctamente a un paciente como enfermo (enfermo positivo); en nuestro estudio fue de $78 \%$. Por otro lado, la especificidad o probabilidad de clasificar a un individuo como sano (sano negativo) fue $44,2 \%$.

El valor predictivo positivo o probabilidad de padecer la enfermedad, a pesar de haber obtenido un resultado positivo en el test, fue de $89,8 \%$. El valor predictivo 
negativo o probabilidad de estar sano a pesar de resultar negativo en la prueba, en nuestro estudio, fue de $24 \%$.

Reyes et al. reportaron una sensibilidad de $89,6 \%$ y especificidad de $69 \%$, el valor predictivo negativo fue de $18,3 \%{ }^{(6)}$. Cedillo et al. tuvieron una sensibilidad de $73 \%$, especificidad de $57 \%$, con valor predictivo positivo (VPP) de $91 \%$, valor predictivo negativo (VPN) de $26 \%{ }^{(8)}$. Por otro lado, Peralta et al. reportan una sensibilidad de $68,9 \%$ y una especificidad de $86,2 \%$ y concluyen que la escala es sensible, pero no específica para el diagnóstico de apendicitis aguda ${ }^{(1)}$.

El diagnóstico clínico mediante la anamnesis, las manifestaciones y el laboratorio constituye, sin lugar a dudas, la herramienta de mayor significancia para la identificación de las enfermedades. La escala de Alvarado emplea estos criterios diagnósticos, acelera la identificación del cuadro clínico y, de esta manera, reduce el tiempo de evolución preoperatorio ${ }^{(10)}$. El análisis con base en curvas ROC, constituye un método estadístico para determinar la especificidad más alta y evaluar la capacidad discriminativa del test diagnóstico, es decir, la capacidad de diferenciar sujetos sanos versus enfermos (11). En nuestro estudio se tuvo un área bajo la curva de 0,611 , lo cual nos indica que es confiable, similar a lo obtenido por Cedillo et al. (2012) que obtuvo 0,748, valor que permite establecer la vigencia de la escala de Alvarado (8). Resultados que difieren de lo hallado por Peralta et al. que encontraron un área bajo la curva de $0,85^{(1)}$, y lo reportado por Reyes et al. quienes obtuvieron el área bajo la curva ROC de $0,89^{(6)}$.

En conclusión, el estudio nos muestra que los criterios utilizados de la escala de Alvarado, son útiles para el diagnóstico de apendicitis aguda, ya que mejoran. Se Este método se emplea todavía y sigue vigente en los servicios de cirugía de tres hospitales de la ciudad del Cusco.

La escala de Alvarado permite el diagnóstico de apendicitis aguda en el grupo de alto riesgo, es un instrumento más para el diagnóstico clínico. Es una herramienta económica y de rápida aplicación en los servicios de urgencia para diagnosticar apendicitis aguda, y permite reducir las apendicectomías negativas, las complicaciones posoperatorias y la disminución de los costos de hospitalización.

\section{REFERENCIAS BIBLIOGRÁFICAS}

1. Peralta KV, Caballero CA, Mora M del P. Validez diagnostica de la escala de Alvarado en pacientes con dolor abdominal sugestivo de apendicitis, en un Hospital de tercer nivel del sur de Bogotá. Rev UDCA Actual Divulg Cient. 2017;20 (1):5-11.

2. Bejarano M, Gallego CXA, Gómez JRO. Frecuencia de abdomen agudo quirúrgico en pacientes que consultan al servicio de urgencias. Rev Colomb Cir. 2011;26 (1):33-41.

3. De Quesada L, Ival M, González CL. La escala de Alvarado como recurso clínico para el diagnóstico de la apendicitis aguda. Rev Cuba Cirugia. 2015;54 (2):121-8.

4. Ricci LE, Ferreyra CM, Córdoba MR, Rios A, Statti MA. Apendicitis aguda segun los criterios de Alvarado. Rev Argent Cirg. 2015;107 (2):57-62.

5. Sanabria Á, Mora M, Domínguez LC, Vega V, Osorio C. Validación de la escala diagnóstica de Alvarado en pacientes con dolor abdominal sugestivo de apendicitis en un centro de segundo nivel de complejidad. Rev Colomb Cir. 2010;25 (1):195-201.

6. Reyes N, Zaldívar FR, Cruz R, Sandoval MD, Gutiérrez CA, Athié C. Precisión diagnóstica de la escala RIPASA para el diagnóstico de apendicitis aguda: análisis comparativo con la escala de Alvarado modificada. Cir Gen. 2012;34 (2):101-6.

7. Hernández L, Dominguez DR. Sensibilidad y especificidad de la escala de Alvarado en apendicitis aguda en el Hospital Regional de Alta Especialidad de Veracruz. Cir Gen. 2012;34 (3):179-84.

8. Cedillo EJ, Santana IA, González R, Onofre J, Gartz GR. Sensibilidad y especificidad de la escala de Alvarado en el diagnóstico de apendicitis aguda comparada con TAC o ultrasonido en las primeras 24 horas de evolución. Cir Gen. 2012;34 (2):107-10.

9. Ospina JM, Barrera LF, Manrique FG. Utilidad de una escala diagnóstica en casos de apendicitis aguda. Rev Colomb. 2011;26 (1):234-41.

10. St Peter SD, Sharp SW, Holcomb GW, Ostlie DJ. An evidencebased definition for perforated appendicitis derived from a prospective randomized trial. J Pediatr Surg. 2008;43(12):2242-5.

11. Cerda J, Cifuentes L. Uso de curvas ROC en investigación clínica: Aspectos teórico-prácticos. Rev Chil Infectol. 2012;29(2):138-41.

Fuentes de financiamiento:

Este artículo ha sido financiado por los autores.

Conflictos de interés:

Los autores declaran no tener ningún conflicto de interés.

\section{Correspondencia:}

Jhuvitza Estela Meléndez Flores

Dirección: Av. Uruguay S/N. Cusco

Teléfono: 927031289

Correo electrónico: itzaela25@gmail.com

Recibido: 24 de julio de 2018 . Evaluado: 30 de julio de 2018. Aprobado: 08 de setiembre de 2018.

(c) La revista. Publicado por Universidad de San Martín de Porres, Perú. (c) By Licencia de Creative Commons Artículo en acceso abierto bajo términos de Licencia Creative Commons Atribución 4.0 Internacional. (http://creativecommons.org/licenses/by/4.0/)

\section{ORCID iDs}

Jhuvitza Estela Meléndez Flores Herbert Cosio Dueñas William Senen Sarmiento Herrera https://orcid.org/0000-0001-6509-3218 https://orcid.org/0000-0002-9981-7576 https://orcid.org/0000-0001-7895-3280 